\title{
$\mathrm{X}-641-68-219$ \\ PREPRINT \\ NASA TM X-63254
}

\section{THE COSMIC GAMMA-RAY SPECTRUM FROM SECONDARY PARTICLE PRODUCTION IN THE METAGALAXY}

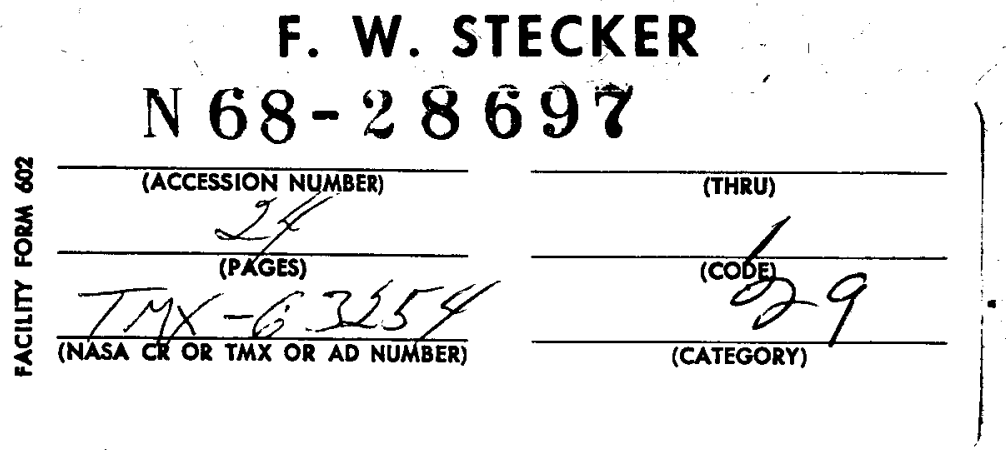

JUNE 1968

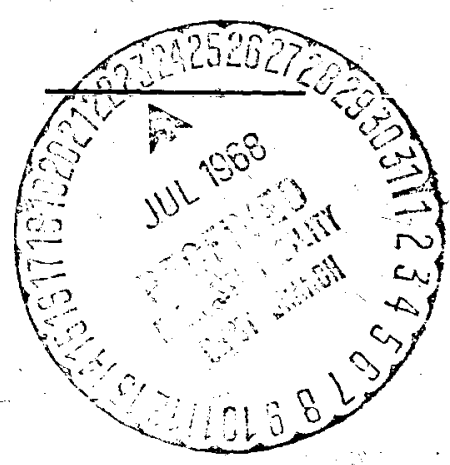


THE COSMIC GAMMA-RAY SPECTRUM

FROM SECONDARY PARTICLE PRODUCTION IN THE METAGALAXY

F. W. Stecker*

June 1968

GODDARD SPACE FLIGHT CENTER

Greenbelt, Maryland

*NRC-NASA Resident Research Associate 
PRECEDING PAGE BLANK NOT, FILMED.

THE COSMIC GAMMA-RAY SPECTRUM

FROM SECONDARY PARTICLE PRODUCTION IN THE METAGALAXY

\author{
F. W. Stecker*
}

\begin{abstract}
The purpose of this paper is to discuss the form and intensity of the spectrum of cosmic gamma-rays resulting from the production and decay of neutral pi-mesons produced in metagalactic cosmic-ray $p-p$ collisions. It is assumed that intergalactic space contains ionized hydrogen gas at a density of $10^{-5} \mathrm{~cm}^{-3}$ as indicated by recent $\mathrm{X}$-ray observations in the $1.5-8 \mathrm{keV}$ region. Using the Friedmann solution to the Einstein field equations of general relativity as a description of our expanding universe, a discussion is presented of the effects of red-shift and spatial curvature on the generation and distortion of the local gamma-ray spectrum from the decay of neutral pi-mesons. Numerical calculations are presented for the Einstein-de Sitter solution, which is found to be an adequate model for these calculations. Various models are presented to represent the possible flux of metagalactic cosmic-rays. In calculating metagalactic gamma-ray spectra, the effect of gamma-ray absorption at large redshifts is taken into account.
\end{abstract}

A discussion of the results is given. The results indicate that future gamma-ray experiments in the $\bar{l}-100 \mathrm{M} e \mathrm{~V}$ region mây yield valuable information

\footnotetext{
"NRC-NASA Resident Research Associate
} 
relating to cosmology, cosmogeny, and the metagalactic cosmic-ray flux. In particular, the metagalactic gamma-ray spectra predicted tend to peak near 70 $\left(1+z_{\max }\right)^{-1} \mathrm{MeV}$ where $z_{\max }$, the maximum red-shift at which cosmic rays are produced, may correspond to the age of the universe at the epoch of galaxy formation. 


\title{
THE COSMIC GAMMA-RAY SPECTRUM
}

\section{FROM SECONDARY PARTICLE PRODUCTION IN THE METAGALAXY}

\author{
F. W. Stecker* \\ Goddard Space Flight Center \\ Greenbelt, Maryland
}

\section{INTRODUCTION}

In recent investigations (Stecker, 1967; Stecker, Tsuruta and Fazio, 1968) the author made use of recent accelerator and cosmic-ray data to determine the details of the cosmic gamma-ray spectrum from the secondary particles produced by cosmic-ray collisions in the galaxy. The purpose of this paper is to determine the cosmic gamma-ray spectrum from secondary particles produced by cosmic-ray collisions in the metagalaxy. This spectrum will differ from the galactic (or local) gamma-ray spectrum because most of the generating collisions take place at large distances where we are looking back to a time when the universe was more compact and collisions were more frequent. These "early" gamma-rays will be of lower energy due to the progressive red-shift of the general cosmic expansion. Although various estimates of the flux of these metagalactic gamma-rays have been made (Ginzburg and Syrovatskii, 1964a, b; Gould and Burbridge, 1965; Garmire and Kraushaar, 1965), none of these workers have takenen cosmological factors into account in order properly to calculate a spectrum.

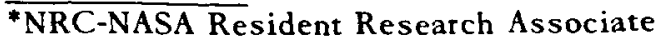




\section{THE COSMOLOGICAL EQUATIONS}

For the purpose of these calculations, we may consider models of the universe which are both homogeneous and isotropic on a large scale. Such models can, in general, be described by the Robertson-Walker line element

$$
d s^{2}=c^{2} d t^{2}-d l^{2}=c^{2} d t^{2}-R^{2}(t) d u^{2}
$$

The time-separable form of the metric is a reflection of the postulated uniformity such that at every moment of world-time the three-space metric is the same at all points and in every direction. Such a three-space is a space of constant Riemannian curvature which may be positive, zero or negative. These three alternatives will be designated by $\mathrm{k}=+1,0,-1$ respectively. If $\mathrm{k}=+1$, the universe is closed and finite; if $\mathrm{k}=0$ the universe is Euclidean, open and infinite; if $\mathrm{k}=-1$ the universe is open, infinite and increasingly divergent in time. More precisely,

$$
\mathrm{du}^{2}=\frac{\mathrm{dr} \mathrm{r}^{2}}{1-\mathrm{kr} \mathrm{r}^{2}}+\mathrm{r}^{2}\left(\mathrm{~d} \theta^{2}+\sin ^{2} \theta \mathrm{d} \phi^{2}\right)
$$

so that du measured along the radial direction is given by

$$
u=\int_{0}^{r} \frac{d r}{\sqrt{1-k r^{2}}}=\left\{\begin{array}{ccl}
\sin ^{-1} r & \text { for } & k=+1 \\
r & \text { for } & k=0 \\
\sinh ^{-1} r & \text { for } & k=-1
\end{array}\right.
$$


Gamma-rays travel along geodesics such that $d s=0$. Assuming a gamma-ray is emitted at a time $t_{e}$ in an interval $\Delta t_{e}$ and received at time $t_{r}$ in an interval $\Delta t_{r}$, it can be shown that

$$
u=c \int_{t_{e}}^{t} \frac{d t}{r(t)}=\text { constant }
$$

and

$$
\frac{c \Delta t_{r}}{c \Delta t_{e}}=\frac{R\left(t_{r}\right)}{R\left(t_{e}\right)}
$$

(For a more detailed discussion of the cosmological relations, the reader is referred to the excellent articles by Sandage referred to in the text.) The time interval, $\Delta \mathrm{t}_{\mathrm{e}}$, is therefore dilated so that

$$
\frac{1}{\Delta t_{e}}=\frac{R\left(t_{r}\right)}{R\left(t_{e}\right)} \frac{1}{\Delta t_{r}}
$$

The gamma-ray is therefore shifted to lower energy by an amount

$$
z=\frac{\Delta \lambda}{\lambda}=\frac{R\left(t_{r}\right)}{R\left(t_{e}\right)}-1
$$


We thus obtain the important relation between red-shift and radius of the universe given by

$$
\frac{R\left(t_{r}\right)}{R\left(t_{e}\right)}=1+z\left(t_{e}\right)
$$

It follows from (6) that in a universe where most of the energy density is in the form of matter

$$
\begin{aligned}
& \frac{n\left(t_{e}\right)}{n\left(t_{r}\right)}=(1+z)^{3} \\
& \frac{T_{\gamma}\left(t_{e}\right)}{T_{\gamma}\left(t_{r}\right)}=(1+z)
\end{aligned}
$$

and

$$
\frac{n_{\gamma}\left(t_{e}\right)}{n_{\gamma}\left(t_{r}\right)}=(1+z)^{3}
$$

where $n(t), T_{\gamma}(t)$ and $n_{\gamma}(t)$ are the average particle density of matter and temperature and photon density of cosmic blackbody radiation in the universe.

We will hereafter designate local $(z=0)$ quantities with a subscript zero. Let $f\left(E_{\gamma}\right)$ be the gamma-ray spectrum generated by the galactic cosmic-ray spectrum, $I_{g}\left(E_{p}\right)$ in traveling a unit particle length $\left(1 \mathrm{~cm}^{-1}\right)$ through the intergalactic medium. (This spectrum is the same as the quantity $I\left(E_{\gamma}\right) /\langle n L\rangle$ cal-culated by Stecker (1967).) 
We now assume that some ubiquitous generating mechanism causes cosmicrays to be produced with the same power law throughout the universe as observed at the earth, so that the metagalactic cosmic-ray spectrum differs only in absolute intensity from the galactic cosmic-ray spectrum. It follows that the form of the cosmic gamma-ray spectrum anywhere in the metagalaxy, when observed in the co-moving frame at that point, will be the same as the form of $f\left(E_{\gamma}\right)$. We may then write down an expression for the integrated metagalactic gammaray flux in any direction as

$$
I\left(E_{\gamma}\right)=\int_{0}^{\ell} \ell_{\max } d \ell n(\ell) \cdot \frac{I(\ell)}{I_{g}} \frac{f\left(E_{\gamma}, \ell\right)}{(1+z(\ell))} e^{-\tau\left(E_{\gamma}, \ell\right)}
$$

where the factor, $(1+z)$, takes into account the reduction in flux due to the time dilation factor and $\mathrm{e}^{-\tau}$ represents absorption of gamma-rays along the line of sight, $I_{g}$ is the galactic cosmic-ray flux and $I(\ell)$ is the cosmic-ray flux at a distance $\ell$. Equation (10) may be put into a much more convenient form by expressing it as an integral over $z$. We then obtain

$$
I\left(E_{\gamma}\right)=\int_{0}^{z_{\max }} \mathrm{d} z \operatorname{nn}(z) \frac{\mathrm{I}(\mathrm{z})}{\mathrm{I}_{\mathrm{g}}} \frac{\mathrm{f}\left(\mathrm{E}_{\gamma}, z\right)}{1+z} \mathrm{e}^{-\tau\left(\mathrm{E}_{\gamma}, z\right)} \frac{\mathrm{d} \ell}{\mathrm{d} z}
$$

Since the energy of a gamma-ray is directly proportional to its frequency, it follows that

$$
f\left(E_{\gamma}, z\right)=f\left[(1+z) E_{\gamma}\right]
$$


It also follows from (7) that

$$
\mathrm{n}(\mathrm{z})=\mathrm{n}_{0}(1+\mathrm{z})^{3}
$$

The quantity

$$
\frac{\mathrm{d} \ell}{\mathrm{d} z}=\mathrm{R}(\mathrm{z}) \frac{\mathrm{du}}{\mathrm{d} z}
$$

depends, in general, both upon the cosmological model involved and the epoch of world-time which defines the acceleration (or deceleration) of the expansion. In Friedmann-type solutions to the Einstein equations, it is found that the expansion of the universe is decelerating. The magnitude of this deceleration is usually denoted by the deceleration parameter q. In the usual notation, the Hubble expansion parameter, $\mathrm{H}$, and the quantity $\mathrm{q}$ are defined by the relations

$$
H \equiv \frac{\dot{R}(t)}{R(t)}
$$

and

$$
q \equiv-\frac{\ddot{R}(t)}{R(t) H^{2}}
$$

In a decelerating universe, therefore, $q>0$. Solutions to the Einstein equation with zero pressure and a cosmological constant of zero may be expressed in 
parametric form in terms of a development angle, $\theta$ (Sandage 1961b) as

$$
\begin{aligned}
& \mathbf{R}=a(1-\cos \theta), \\
& t=\frac{a}{c}(\theta-\sin \theta) .
\end{aligned}
$$

for $\mathrm{k}=+1$, and

$$
\begin{aligned}
& \mathbf{R}=\mathrm{a}(\cosh \theta-1), \\
& \mathbf{t}=\frac{\mathrm{a}}{\mathrm{c}}(\sinh \theta-\theta),
\end{aligned}
$$

for $\mathrm{k}=-1$, where $\mathrm{a}=4 \pi \mathrm{G} \rho \mathbf{R}^{3} / 3 \mathrm{c}^{2}$ and $\rho$ is the density of matter in the universe (so that $\rho \mathbf{R}^{3}=$ constant).

For the Euclidean case of $k=0, R(t)$ can be expressed explicitly in terms of $t$ by the relation

$$
R(t)=\left(6 \pi \mathrm{G} \rho R^{3}\right)^{1 / 3} t^{2 / 3}
$$

From (15) and (18) it then follows that for $k=0$ that

$$
q=\frac{1}{2} \text { for all } t \text {. }
$$

For $\mathrm{k}=+1$, it follows from (15) from (16) that

$$
\mathrm{q}=\frac{1-\cos \theta}{\sin ^{2} \theta}
$$


For $k=-1$, it follows from (15) and (17) that $q$ is given by

$$
q=\frac{1-\cosh \theta}{\sinh ^{2} \theta}
$$

For $\theta<1$, corresponding to an early epoch of the expansion, (20) and (21) both reduce to the Euclidean case of $q=1 / 2$. The Euclidean (Einstein-de Sitter) model is therefore a good approximation to the univer se if it has not yet reached a highly evolved state. It is also compatable with the most probable values of $q$ as discussed by Sandage (1961a, 1962), based on the observed magnitude-red shift relation, and with the recent determination by Henry, Fritz, Meekins, Friedman and Byram (1968) of a mean metagalactic gas density of the order of $10^{-5} \mathrm{~cm}^{-3}$

Under the assumption of a Euclidean model, we will now determine the cosmological effects on the metagalactic gamma-ray spectrum. It can be shown (Sandage 1961b) that

$$
\frac{\mathrm{d} \ell}{\mathrm{d} z}=\frac{\mathrm{cH}_{0}^{-1}}{(1+z)^{2}\left(1+2 \mathrm{q}_{0} \mathrm{z}\right)^{1 / 2}}
$$

where $\mathrm{cH}_{0}^{-1}=10^{28} \mathrm{~cm}$. In the Euclidean case, $\mathrm{q}_{0}=1 / 2$ and we may take in equation (11)

$$
\frac{\mathrm{d} \ell}{\mathrm{dz}}=\frac{10^{28}}{(1+z)^{5 / 2}}
$$




\section{ABSORPTION OF METAGALACTIC GAMMA-RAYS}

An excellent discussion of the absorption processes affecting cosmic gammarays has been given by Fazio (1967). The principal absorption process to be considered is that of electron-positron pair production through interaction with the universal black-body radiation field, i.e., the reaction

$$
y+\gamma \rightarrow \mathrm{e}^{+}+\mathrm{e}^{-}
$$

Detailed calculations of the energy-dependent absorption probability for this process have been performed by Gould and Schréder (1967). They have shown that for a gamma-ray of energy $E_{\gamma}$ interacting with a black-body radiation field of temperature $\mathrm{T}_{\gamma}$

$$
\frac{\mathrm{d} \tau}{\mathrm{d} \ell} \simeq \frac{a^{2}}{2 \pi \Lambda}\left(\frac{\mathrm{kT}_{\gamma}}{\mathrm{mc}^{2}}\right)^{3} \sqrt{\xi} \mathrm{e}^{-\xi}
$$

where

$$
\xi \equiv \frac{\left(\mathrm{mc}^{2}\right)^{2}}{\mathrm{kT}_{\gamma} \mathrm{E}_{\gamma}}>>1
$$

where $\alpha \simeq 1 / 137$ is the fine-structure constant, $\Lambda=\hbar / \mathrm{mc}=3.86 \times 10^{-11} \mathrm{~cm}$, and $\mathrm{k}$ here is Boltzmann's constant. The local black-body temperature has been found by Stokes, Partridge and Wilkinson (1967) to be

$$
\mathrm{T}_{0} \square 2.7^{\circ} \mathrm{K}
$$


so that the condition $\xi>>1$ corresponds to the condition

$$
\mathrm{E}_{\gamma}<\frac{1.12 \times 10^{6} \mathrm{GeV}}{(1+z)^{2}}
$$

(see equation (8) and (12)).

We will restrict ourselves here to a determination of the gamma-ray spectrum below $1 \mathrm{GeV}$ and $z \leq 10^{3}$ (as will be discussed later) so that the approximation given by equation (25) will be generally valid. Therefore, from (23) and (25), we find

$$
\tau\left(E_{\gamma}, z\right)=3.9 \times 10^{8} E_{\gamma}^{-1 / 2} \int_{0}^{z} \mathrm{dy} \frac{\exp \left[-\frac{1.12 \times 10^{6} \mathrm{E}}{(1+\mathrm{y})^{2} \mathrm{E}_{\gamma}}\right]}{(1+\mathrm{y})^{1 / 2}}
$$

(See appendix for further discussion.)

\section{THE METAGALACTIC COSMIC-RAY SPECTRUM}

It now remains only to specify a suitable model for the metagalactic cosmic ray flux. We will as sume that at some early epoch, corresponding to $z \geq z_{\max }$ conditions were unsuitable for the acceleration of cosmic-rays. We will consider $z_{\max }$ to correspond to the epoch of galaxy formation and consider two possible models for the origin of a metagalactic cosmic-ray flux. For model I, we will assume that the metagalactic flux arises through a constant leakage rate from the halos of galaxies from $z=z_{\max }$ to $z=0$. For model II, we will assume 
that this flux was created primarily in a burst at the time of galaxy formation. Thus, model I and model II correspond to the two extreme cases which may be expected.* For $z_{\max }$, we will also consider two extremes. One extreme is $z_{\max }=10^{3}$, which corresponds to the earliest epoch when galaxy formation could probably occur. At $z=10^{3}$, the black-body temperature of the universe was of the order of $10^{3}-10^{4} \mathrm{~K}$, cool enough for ionized hydrogen to combine to form a neutral gas. According to Peebles (1965), $z=10^{3}$ also corresponds to the epoch when gas clouds may begin to form gravitationally bound systems.

The other extreme for $z_{\max }$ which we may consider corresponds to the highest red-shift yet observed for a quasar, vis., 2.2. This is, of course, an extreme which is technique-limited rather than being limited by any physical criteria, and it is included mainly for purposes of discussion. We will also consider various intermediate values for $\mathrm{z}_{\max }$ of 4,9 , and $10^{2}$. (Doroshkevich, et. al. (1967) suggest that galaxy formation took place at $z=10-20$ whereas Weymann (1967) suggests $z=10^{2}$.)

It is important to note here that the upper limit, $z_{\max }$, may be effectively restricted, not by the epoch of galaxy formation, but by attenuation of the metagalactic cosmic-ray flux due to the collisions themselves. The cross-section for inelastic cosmic-ray $p-p$ collisions is of the order of $30 \mathrm{mb}$. Therefore, the lifetime of the metagalactic cosmic-rays against collisional losses is given

\footnotetext{
*Models allowing for the possibility of increased cosmic-ray production by galaxies in the past, as well as other possibilities, will be considered in a future paper. In particular, it may be of interest to examine the possibility that the isotropic $X$-ray spectrum may be a red-shifted $\pi^{\circ}$-gammaray spectrum, a hypothes is which may explain both the intensity and power-law form of the spectrum.
} 
by

$$
\begin{aligned}
\tau_{\mathrm{c}}=\frac{1}{\mathrm{n} \sigma \mathrm{c}} & =\frac{1}{\mathrm{n}_{0} \sigma \mathrm{c}(1+\mathrm{z})^{3}} \\
& \simeq 10^{20}(1+\mathrm{z})^{-3} \mathrm{sec}
\end{aligned}
$$

for $\mathrm{n}_{0}=10^{-5} \mathrm{~cm}^{-3}$.

The lifetime of the universe at a red-shift $\mathrm{z}$ is given by

$$
\tau_{u} \simeq 10^{17}(1+z)^{-3 / 2} \mathrm{sec}
$$

Cosmic-rays cannot accumulate in the metagalaxy if the ratio, $\tau_{\mathrm{c}} / \tau_{\mathrm{u}}<1$.

The condition $\tau_{c} / \tau_{u}=1$ therefore defines a critical value of $z_{\max }=10^{2}$ beyond which a further buildup of metagalactic cosmic-rays cannot occur. With these limitations on $z$ in mind, we will now consider the various ideal models for describing the metagalactic cosmic-ray flux.

Model I: In this model, we assume that the galaxies were created on the order of $10^{10}$ years ago, as indicated by stellar evolution studies in our own galaxy. We will assume that ours is a typical galaxy for which the leakage time of cosmic rays from the halo is on the order of $10^{8}$ years. Therefore each galaxy has emitted about $10^{2}$ halo-volumes of cosmic-rays since the time of galaxy formation. The ratio of the volume of one galactic halo to the volume of 
the universe is

$$
\frac{V_{\mathrm{g}}}{\mathrm{V}_{\mathrm{u}}} \simeq\left(\frac{5 \times 10^{22} \mathrm{~cm} .}{10^{28} \mathrm{~cm} .}\right)^{3} \simeq 10^{-16}
$$

Taking the cosmic-ray flux in our galaxy as an average for all galaxies and taking the number of galaxies in the universe to be $3 \times 10^{9}$ (Allen, 1963), we find that

$$
I_{0} \simeq 10^{-16} \times 3 \times 10^{9} \times 10^{2} \mathrm{I}_{\mathrm{g}}=3 \times 10^{-5} \mathrm{I}_{\mathrm{g}}
$$

We assume a constant leakage rate so that the total number of cosmic-rays in the metagalaxy is proportional to the time elapsed since galaxy formation. It follows from (18) that this time is given by

$$
\tau_{\mathrm{g}} \simeq 10^{10}\left[(1+z)^{-3 / 2}-\left(1+z_{\max }\right)^{-3 / 2}\right] \mathrm{yrs}
$$

The cosmic-ray density will then increase with red-shift according to the relation

$$
\frac{I^{\prime}(g)}{I_{g}} \simeq 3 \times 10^{-5}(1+z)^{3}\left[(1+z)^{-3 / 2}-\left(1+z_{\max }\right)^{-3 / 2}\right]
$$

However, the cosmic-rays which produce the neutral pi-mesons necessary for gamma-ray production are only those above a threshold energy, $E_{t h}$, of about 
$300 \mathrm{MeV}$ (Stecker, 1966). We must therefore determine

$$
I(z)=I^{\prime}\left(E>E_{t h} ; z\right)
$$

For a power low cosmic-ray spectrum of the form

$$
\mathrm{I}(>\mathrm{E}) \sim \mathrm{E}^{-3 / 2}
$$

it follows from the red-shift relation that

$$
\begin{aligned}
I\left(E>E_{t h} ; z\right) & =I^{\prime}\left(E>\frac{E_{t h}}{(1+z)}\right) \\
& =I^{\prime}(z)\left[\frac{1+z}{1+z_{\max }}\right]^{3 / 2}
\end{aligned}
$$

so that we must use an effective flux of

$$
\frac{I(z)}{I_{g}} \simeq 3 \times 10^{-5}(1+z)^{3}\left[\frac{1+z}{1+z_{\max }}\right]^{3 / 2}\left[(1+z)^{-3 / 2}-\left(1+z_{\max }\right)^{-3 / 2}\right]
$$

Model II: In this model we assume that the metagalactic cosmic-rays were created in a burst at the time of galaxy formation. Assuming that the total number of cosmic-rays released in the initial burst is equal to the total number released over $10^{10}$ years in model $\mathrm{I}$, we find

$$
\frac{I(z)}{I_{g}}=3 \times 10^{-5}(1+z)^{3}\left[\frac{1+z}{1+z_{\max }}\right]^{3 / 2}
$$


Model III: For a final comparison, we compute the integrated gamma-ray flux generated in the galaxies themselves and determine their contribution to the metagalactic-gamma-ray flux. We take the average amount of matter in galaxies to be about $1 \%$ of the total matter in metagalactic space and assume that on the average, a fraction of $5 \times 10^{-2}$ of this matter is in the form of gas (Allen, 1963; Roberts, 1963). Then taking $I(z) \simeq I_{g}$, we find that in this case

$$
\frac{\langle I(z) n(z)\rangle}{I_{g} n_{0}}=5 \times 10^{-4}(1+z)^{3}
$$

Using the models defined by equations (34), (38), (39) and (40), together with equations $(11),(12),(13),(23)$ and $(28)$, we have calculated the metagalactic gamma-ray spectra produced by models I, II and III. These fluxes are given in Figures 1, 2 and 3 respectively. Figures $1-3$ also show the gamma ray flux expected from the galactic halo in the direction of the pole, taking $\langle\mathrm{nL}\rangle=3 \times 10^{20}$ $\mathrm{cm}^{-2}$ and based on previous calculations (Stecker, 1967).* It can be seen that the local gamma-ray spectrum from the galactic halo can be distinguished from the metagalactic gamma-ray spectra because the latter are red-shifted and peak at lower energies. The metagalactic gamma-ray spectra tend to peak near $7 \times 10^{-2} /$ $\left(1+z_{\max }\right) \mathrm{GeV}$, being weighted toward higher red-shifts by the effect of greater densities at earlier epochs. Because of the density effect, a cosmic-ray burst

*Thus $\mathrm{I}_{\text {pole }}\left(\mathrm{E}_{\gamma}\right)=3 \times 10^{20} \mathrm{f}\left(\mathrm{E}_{\gamma}\right)$. 
at large red-shifts is much more effective in producing gamma-rays then a continuous production of the same number of cosmic-rays. In Figures 1 - 3, we have also indicated the experimental upper limit on the gamma-ray flux from the Explorer XI data (Kraushaar, Clark, Garmire, Helmkin, Higbie and Agogino, 1965), assuming an integral flux above $0.1 \mathrm{GeV}$ of $3 \times 10^{-4} \mathrm{~cm}^{-2} \mathrm{sec}^{-1}$ $\mathrm{sr}^{-1}$

\section{CONCLUSIONS}

Present evidence about the flux of cosmic-rays between the galaxies is quite meager. The most promising way to study the flux is by a satellite experiment measuring the isotropic gamma-ray flux in the region between 1 and $100 \mathrm{MeV}$. Such gamma-rays can supply us with direct information on metagalactic cosmic-rays, because they travel to us in straight lines and suffer little absorption. Theoretical metagalactic gamma-ray fluxes from $\pi^{0}$ decay are presented here under various assumptions as to the metagalactic cosmic-ray flux. These predictions indicate that an experimental determination of the isotropic gamma-ray spectrum at high galactic latitudes and in the energy range $1-100$ $\mathrm{MeV}$, could supply valuable information, not only about metagalactic cosmic-rays, but also about such fundamental questions as when the galaxies were formed, since the metagalactic gamma-ray spectrum will peak near $70\left(1+z_{\max }\right)^{-1} \mathrm{MeV}$. 


\section{ACKNOWLEDGMENTS}

The author is indebted to Dr. Richard C. Henry of the Naval Research Laboratory for supplying him with the details of the NRL X-ray observations prior to publication and for several stimulating discussions. The author would also like to acknowledge and thank Dr. Frank C. Jones for his comments and Mr. Joseph Bredekamp for programming the numerical calculations essential to this paper.

\section{REF ER ENCES}

Allen, C. W., 1963, Astrophysical Quantities, 2nd. ed., Athlone Press, London. Doroshkevich, A. G., Zel'dovich, Y. B., and Novikov, I. D., 1967, Sov. Ast. A. J., 11,233 .

Fazio, G. G., 1967, Ann. Rev. Astron. and Ap., 5 , 481.

Garmire, G. and Kraushaar, W., 1965, Space Sci. Rev., 4, 123.

Ginzburg, V. L. and Syrovatskii, S. I., 1964a, Sov. Phys. JETP, 18, 245.

Ginzburg, V. L. and Syrovatskii, S. I., 1964b, The Origin of Cosmic Rays, Macmillan Co., New York.

Gould, R. J. and Burbidge, G. R., 1965, Ann. d'Astrophys., 28, 171.

Gould, R. J. and Schréder, G. P., 1967, Phys. Rev., 155, 1404.

Henry, R. C., Fritz, G., Meekins, J. F., Friedman, H., and Byram, E. T., preprint.

Kraushaar, W., Clark, G. W. Garmire, G., Helmkin, H., Higbie, P. and Agogino, M., 1965, ApJ., 141, 845 . 
Peebles, P. J. E., 1965, 142, 1317.

Roberts, M. S., 1963, Ann. Rev. Astron. and Ap., 1, 149.

Sandage, A., 1961a, ApJ., 133, 355.

Sandage, A., 1961b, ApJ., 134, 916.

Sandage, A., 1962, J. Soc. Indus. Appl. Math, 10, 781.

Stecker, F. W., 1966, Smithsonian Ap. Obs. Spec. Rept. No. 220.

Stecker, F. W., 1967, Smithsonian Ap. Obs. Spec. Rept. No. 260.

Stecker, F. W., Tsuruta, S., and Fazio, G. G., 1968, ApJ., 151, 881.

Stokes, G. A., Partridge, R. B., and Wilkinson, D. T., 1967, Phys. Rev. Letters, 19,1199 。

Weymann, R., 1967, ApJ., 147, 887. 


\section{APPENDIX \\ GAMMA-RAY OPACITY IN AN EINSTEIN-DE SITTER UNIVERSE}

It has been shown in the text that the gamma-ray absorption from pair production through interaction with the universal radiation field is given by the factor $\mathrm{e}^{-\tau}$ where

$$
\tau\left(E_{\gamma}, z\right)=3.9 \times 10^{8} E_{\gamma}^{-1 / 2} \int_{0}^{z} \mathrm{dy}(1+y)^{-1 / 2} \exp \left[-\frac{1.12 \times 10^{6}}{(1+y)^{2} E_{\gamma}}\right](A-1)
$$

with $\mathrm{E}_{\gamma}$ expressed in $\mathrm{GeV}$. As an intermediate solution to the problem considered in the text, numerical solutions where obtained for the implicit relation

$$
\tau\left(\mathbf{E}_{\gamma}, \mathbf{z}_{\gamma}\right) \equiv \mathbf{1}
$$

which defines the red-shift, $z_{\gamma}$, beyond which the universe becomes opaque to gamma-rays of local energy $\mathrm{E}_{\gamma^{*}}$. It was found that the numerical solution to equation (A-2) may be quite well approximated by the relation

$$
1+z_{\gamma} \simeq 2.30 \times 10^{2} \mathrm{E}_{\gamma}^{-0.484}
$$

Since the age of the universe corresponding to a red-shift $z_{\gamma}$, is given by

$$
t_{\gamma} \simeq 10^{10}\left(1+z_{\gamma}\right)^{-3 / 2} \text { years }
$$


the earliest epoch from which gamma-rays of energy $\mathbf{E}_{\gamma}$ can supply us with information is found from $(A-3)$ and $(A-4)$ to be

$$
\mathbf{t}_{\gamma}=2.9 \times 10^{6} \mathrm{E}_{\gamma}^{3 / 4} \text { years }
$$




\section{FIGURE CAPTIONS}

Figure 1: Metagalactic gamma-ray spectra from cosmic-ray p-p interactions based on a cosmic-ray flux produced by constant leakage from other galaxies (Model I) and shown for various maximum red-shifts as discussed in the text. Also shown are the local gamma-ray spectrum from the galactic halo in the direction of the pole and the upper limit implied by the Explorer XI gamma-ray experiment.

Figure 2: Metagalactic gamma-ray spectra from cosmic-ray p-p interactions based on a cosmic-ray flux produced by a burst of cosmic rays at $z_{\max }$ (Model II) as discussed in the text. Also shown are the local gamma-ray spectrum from the galactic halo in the direction of the pole and the upper limit implied by the Explorer XI gamma-ray experiment.

Figure 3: Metagalactic gamma-ray spectra from the superposition of gammaray spectra produced in all the galaxies taking red-shift, density and curvature effects into account as explained in the text (Model III). Also shown are the local gamma-ray spectrum from the galactic halo in the direction of the pole and the upper limit implied by the Explorer XI gamma-ray experiment. 


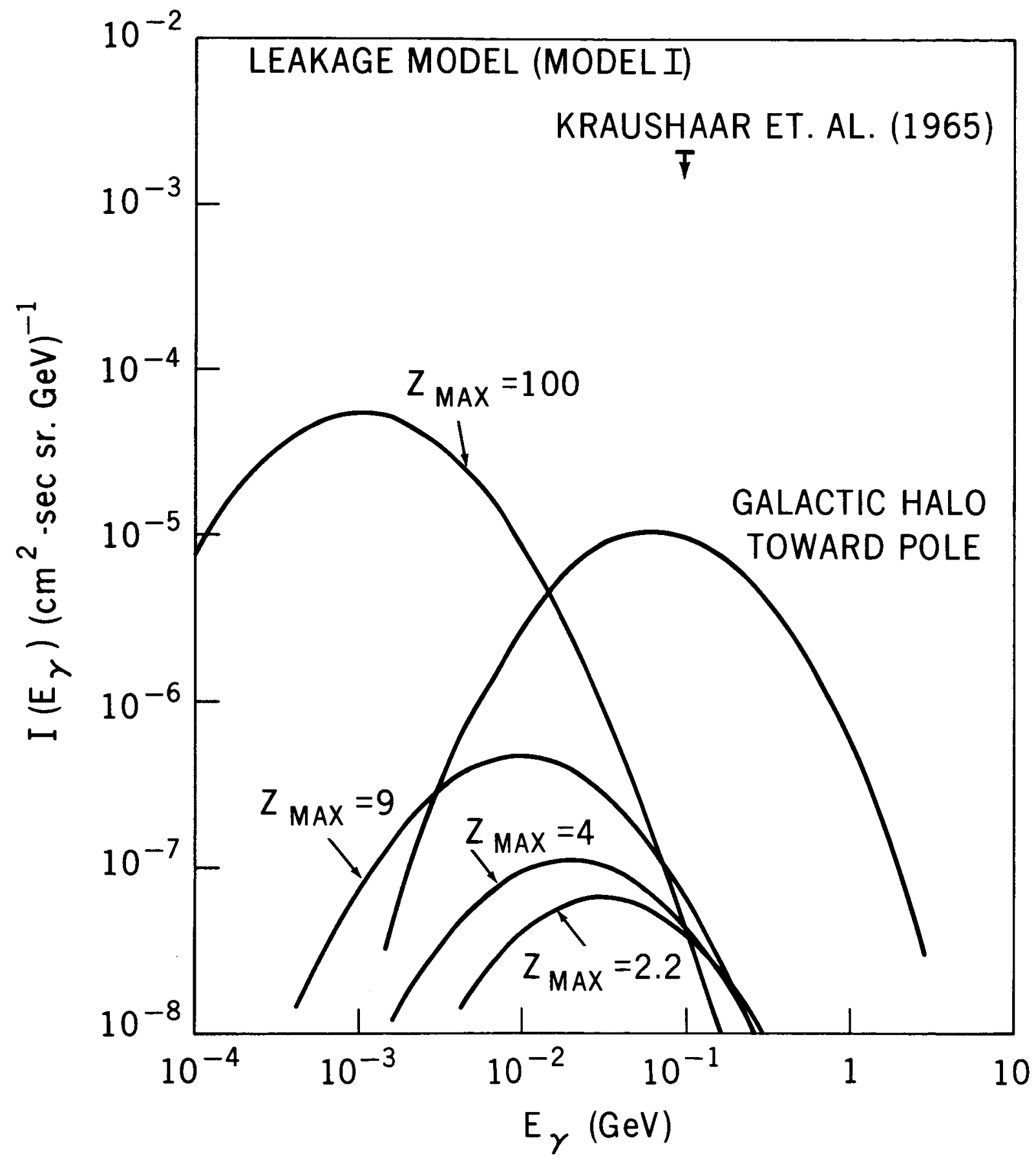




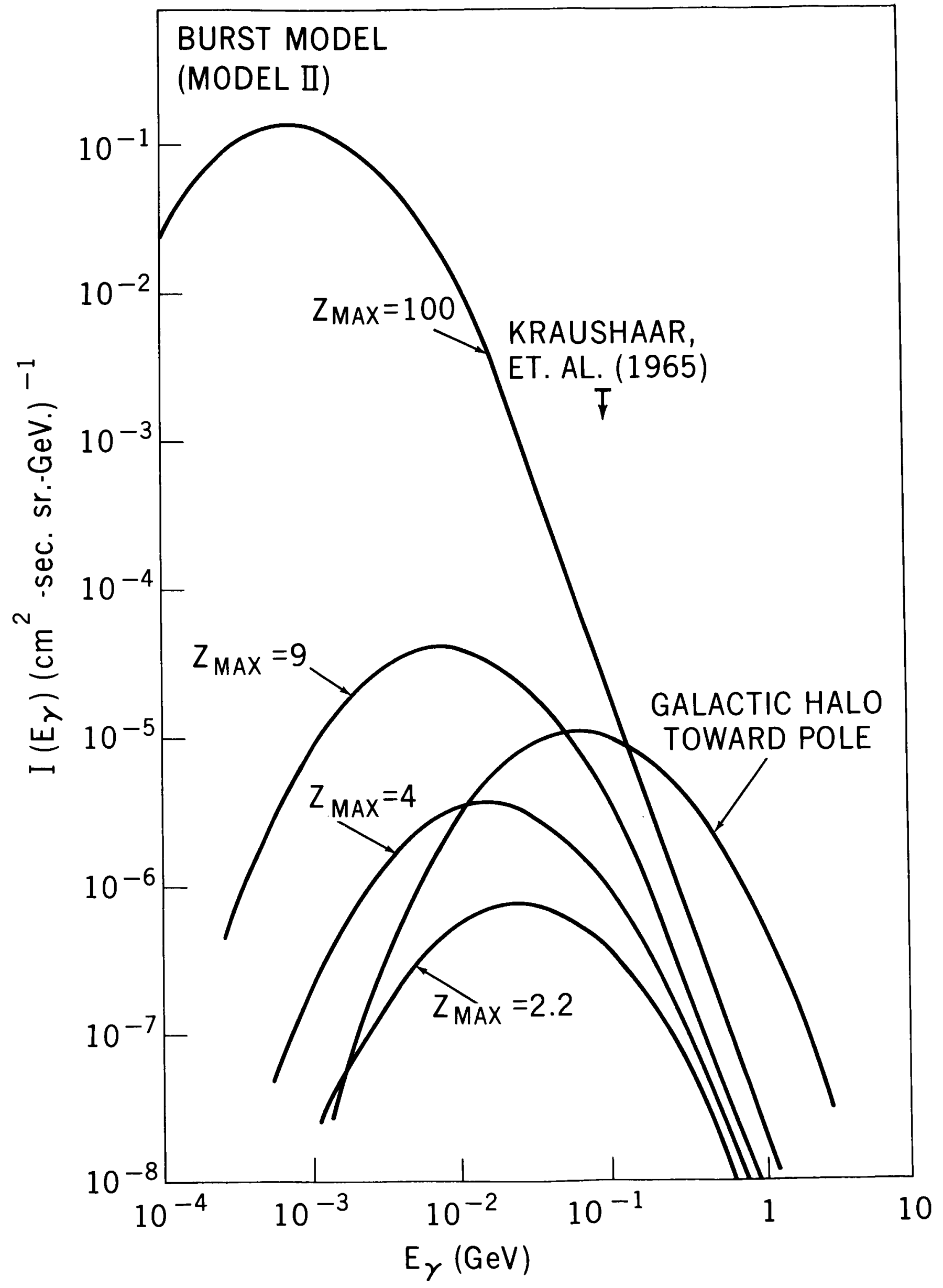




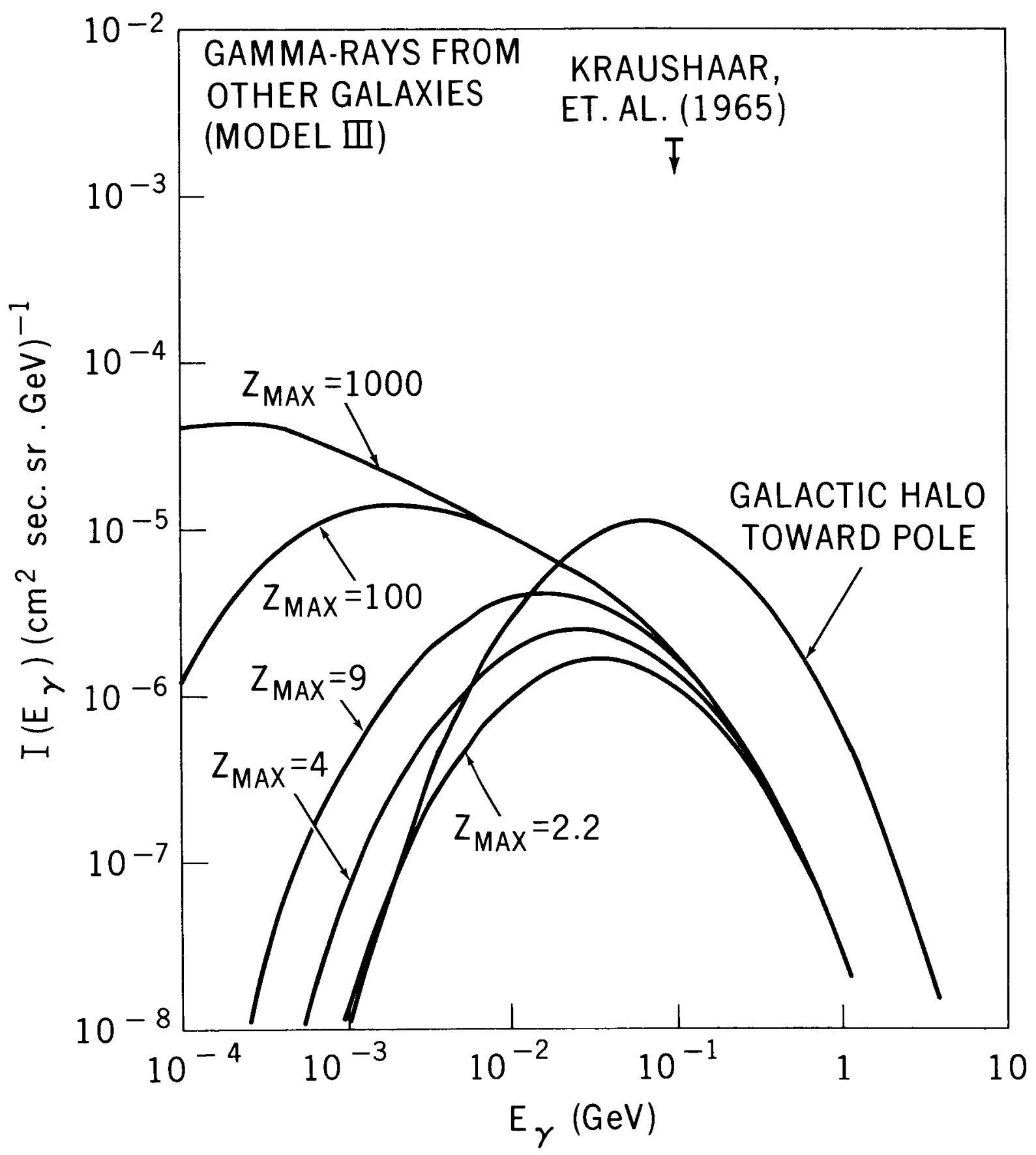

